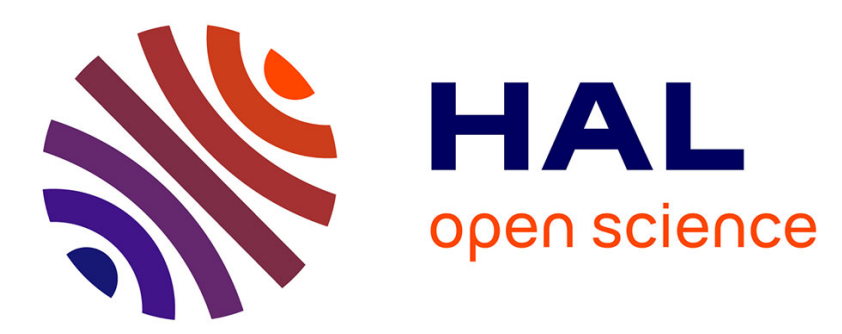

\title{
Scaling and density of states of fractal lattices from a generating function point of view
}

\author{
A.-M.S. Tremblay, B.W. Southern
}

\section{To cite this version:}

A.-M.S. Tremblay, B.W. Southern. Scaling and density of states of fractal lattices from a generating function point of view. Journal de Physique Lettres, 1983, 44 (20), pp.843-852. 10.1051/jphyslet:019830044020084300 . jpa-00232272

\section{HAL Id: jpa-00232272 https://hal.science/jpa-00232272}

Submitted on 1 Jan 1983

HAL is a multi-disciplinary open access archive for the deposit and dissemination of scientific research documents, whether they are published or not. The documents may come from teaching and research institutions in France or abroad, or from public or private research centers.
L'archive ouverte pluridisciplinaire HAL, est destinée au dépôt et à la diffusion de documents scientifiques de niveau recherche, publiés ou non, émanant des établissements d'enseignement et de recherche français ou étrangers, des laboratoires publics ou privés. 
Classification

Physics Abstracts

$64.70-63.50-71.10-72.90$

\title{
Scaling and density of states of fractal lattices from a generating function point of view
}

\author{
A.-M. S. Tremblay \\ Département de Physique, Université de Sherbrooke, Sherbrooke, Québec J1K 2R1, Canada \\ and B. W. Southern
}

Department of Physics, University of Manitoba, Winnipeg, Manitoba R3T 2N2, Canada

(Reçu le 2 mai 1983, révisé le 26 juillet, accepté le 2 septembre 1983)

\begin{abstract}
Résumé. - Il est démontré que l'analogie entre l'énergie libre des phénomènes critiques et la fonction génératrice complexe rend possible l'utilisation de techniques bien connues du groupe de renormalisation dans l'espace direct, permettant ainsi de dériver facilement les propriétés d'échelle ainsi que la densité d'états exacte pour des problèmes électroniques ou de vibration sur des réseaux fractals. On discute aussi l'existence d'une famille de réseaux dont la dimension spectrale $\tilde{d}$ peut être inférieure ou supérieure à la valeur 2 .
\end{abstract}

\begin{abstract}
It is shown that the analogy between the free energy in critical phenomena and the complex generating function allows one to exploit well known position-space renormalization group techniques to easily derive scaling properties as well as the exact density of states for electronic or vibrational problems on fractal lattices. Certain self-similar lattices whose spectral dimension $\tilde{d}$ can be larger or less than 2 are also studied.
\end{abstract}

Self-similar (or fractal [1]) lattices have, for the past few years, been the subject of much attention in the Physics community. The structure of fractal lattices suggests that they may serve as simple models for disordered systems such as polymers or percolation clusters near threshold [2]. These lattices lack translational invariance but possess dilation symmetry, making the renormalization group an ideal tool to study their properties.

The phase transition properties of fractal lattices have thus been the first to attract attention [3, 4]. Gefen et al. [4] have emphasized that the universality class to which a given lattice belongs is determined not only by the topological dimensionality $d$ and by the fractal dimensionality $\bar{d}$, but also by the order of ramification and other properties of the lattice. The recognition by Berker and Ostlund [5] that approximate Migdal-Kadanoff recursion relations become exact for certain self-similar hierarchical lattices, certainly justifies even more investigations of fractal lattices. It should also be pointed out that, in general, a large category of problems on fractal lattices may be exactly solved by decimation techniques [6] which can otherwise be implemented exactly only in one dimension.

Recently, four groups [7-12] have studied the " spectral properties " of such lattices, e.g., the density of states (DOS) of a tight-binding electronic problem or of a vibration problem, or the 
problem of a random walk on such lattices. (It has also been pointed out recently [13] that the local density of states on certain sites of a hierarchical lattice may serve as a model for disordered one-dimensional systems). Alexander and Orbach [7] and especially Rammal and Toulouse [9] have emphasized that for fractal lattices, one should distinguish the fractal dimension $\bar{d}$, that of the embedding Euclidean space $d$ and the spectral dimension $\tilde{d}$ (to be defined below). Most of the analysis of these two groups is based on a scaling hypothesis. Rammal and Toulouse [8], Domany et al. [10] and Rammal [11,12] have performed actual computations of the DOS for various cases. They found that the DOS has a complicated structure and that scaling in this case implies self-similarity but not power law behaviour. Although elegant, the actual technique used by all these groups, except Rammal [11, 12], is very cumbersome, mainly because the recursion relations are singular for certain values of the energy, which in their case can take only real values

In the present paper, we want to exploit more fully the analogies with critical phenomena to explore the spectral properties of fractal lattices. (Rammal, Ref. 12, has done parallel work on this subject). More specifically, we wish to show that if one is willing to work with the complex generating function which is the analog of a free energy, one obtains non-singular recursion relations [13, 14], as well as an analytical proof of scaling and a very simple way to evaluate the DOS. We give examples of numerical calculations of the DOS for the Sierpinsky lattices in Euclidean $d=2$ and $d=3$. In the last section, we consider Berker-Ostlund hierarchical lattices which, as we show, may have spectral dimension $\tilde{d}$ larger than 2 , in contrast with the Sierpińsky lattices in arbitrary dimension [9] which have $\tilde{d}$ less than or equal to 2 . We also demonstrate that these lattices may have continuously varying $\tilde{d}$, including $\tilde{d}<2$ and $\tilde{d}>2$, a property which may have some relevance to the problem of localization [9].

\section{Complex generating function and examples of numerical calculation of the density of states on fractal lattices.}

To be more specific, let us restrict ourselves to an electronic tight-binding problem. If $\varepsilon$ is the single site energy and $V$ the nearest-neighbour hopping matrix element, the Green's function equations for that problem are, in general,

$$
(E+i \eta-\varepsilon) G_{i j}=\delta_{i j}-\sum_{n} V G_{i+n j}
$$

where $n$ labels the nearest-neighbours, $E$ is the energy parameter and $i \eta$ is a pure imaginary number.

We first recall known facts about the generating function and then proceed with applications to fractals. Define the row vector

$$
U^{\mathrm{T}} \equiv\left(u_{1}, u_{2}, \ldots, u_{N}\right)
$$

where $u_{i}$ are continuous variables on each site of the lattice, and define the matrix $\mathbf{H}$ such that $\operatorname{det}(E \mathbf{I}-\mathbf{H})=0$ when $E$ is an eigenvalue (I is the identity matrix). Then it is not too difficult to show that $[13,15,16]$

with

$$
\mathcal{F}=\ln \left\{\int \mathfrak{D} u \exp \left[\frac{i}{2} U^{\mathbf{T}}((E+i \eta) \mathbf{I}-\mathbf{H}) U\right]\right\}
$$

$$
\int \mathfrak{D} u \equiv \prod_{i=1}^{N} \int_{-\infty}^{\infty} \mathrm{d} u_{i}
$$

is the generating function for this problem. Note that the small positive number $\eta$ ensures conver- 
gence of the integrals. We need the following identity for the function $\rho(E, \eta)$. Let $E_{\alpha}$ be an eigenvalue, then [15],

$$
\begin{aligned}
\rho(E, \eta) & =\frac{1}{N} \frac{2}{\pi} \operatorname{Im}\left(\frac{\partial \mathcal{F}(E+i \eta)}{\partial E}\right) \\
& =\frac{1}{N} \frac{1}{\pi} \sum_{\alpha} \frac{\eta}{\left(E-E_{\alpha}\right)^{2}+\eta^{2}}
\end{aligned}
$$

which may easily be proven by performing an orthonormal transformation in equation 2 to a basis where $\mathbf{H}$ is diagonal. In the limit $\eta \rightarrow 0$, the function $\rho(E, \eta)$ becomes the true density of states. With $\eta$ finite but small, we can still call $\rho(E, \eta)$ the DOS : the value of $\eta$ then determines the precision with which each eigenvalue can be obtained from that DOS.

To compute $\mathcal{F}$ and hence the DOS of a general lattice or of a fractal lattice in particular, one proceeds as follows. Let 2 symbolically represent all the indices which label the sites on the smallest length scale, and let 1 stand for the other sites. Following the renormalization group prescription, one integrates over all sites 2 contained in the generating function and then one defines a new generating function with renormalized parameters such that it is equal to the old generating function. One then finds that within an energy independent term which does not contribute to the DOS in equation $3, \mathcal{F}$ becomes,

$$
\mathscr{F}=\ln \left\{\int \mathfrak{D} u^{\prime} \exp \left[\frac{i}{2} U^{\prime T}\left((E+i \eta) \mathbf{I}^{\prime}-\mathbf{H}_{11}^{\prime}\right) U^{\prime}\right]\right\}+N C^{\prime}
$$

where the integral is now over only the uneliminated sites and where

$$
\begin{aligned}
& \mathbf{H}_{11}^{\prime}=\mathbf{H}_{11}+\mathbf{H}_{12}\left[(E+i \eta) \mathbf{I}-\mathbf{H}_{22}\right]^{-1} \mathbf{H}_{21} \\
& N C^{\prime}=-\frac{1}{2} \ln \left\{\operatorname{det}\left[(E+i \eta) \mathbf{I}-\mathbf{H}_{22}\right]\right\} .
\end{aligned}
$$

As an example, let us consider first the Sierpińsky gasket illustrated in figure 1. As found in references 9 and 10, one finds that the decimation procedure described above does not lead to longer range hopping and hence may easily be implemented. One obtains here three complex, non-singular recursion relations. One for $E+i \eta-\varepsilon$, one for $V$ and one for the $u$ independent term (so called " constant term ") $N C^{\prime}$ in equations 5 and $6 b$. Upon iteration, $V$ goes to zero at a rate which depends in particular on the value of $\eta$. Along the trajectory to this fixed point, one can accumulate the value of the constant term to finally obtain $\mathcal{F}$ and hence the DOS from equation 2. For initial values $V=1$ and $\varepsilon=0$, which we use throughout, one obtains the spectrum

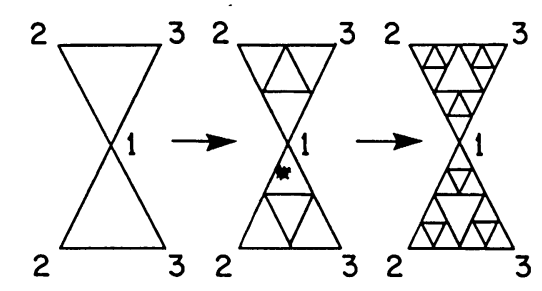

Fig. 1. - Construction of the Sierpińsky gasket. At each level, one inserts three sites in each of the triangles and joins them by bonds to obtain the next level. With periodic boundary conditions, the sites $1,2,3$ on the largest two triangles are identified. Equation 1 applies to the bonds and sites at the smallest length scale. The decimation procedure starts by elimination of the sites on the smallest scale. 


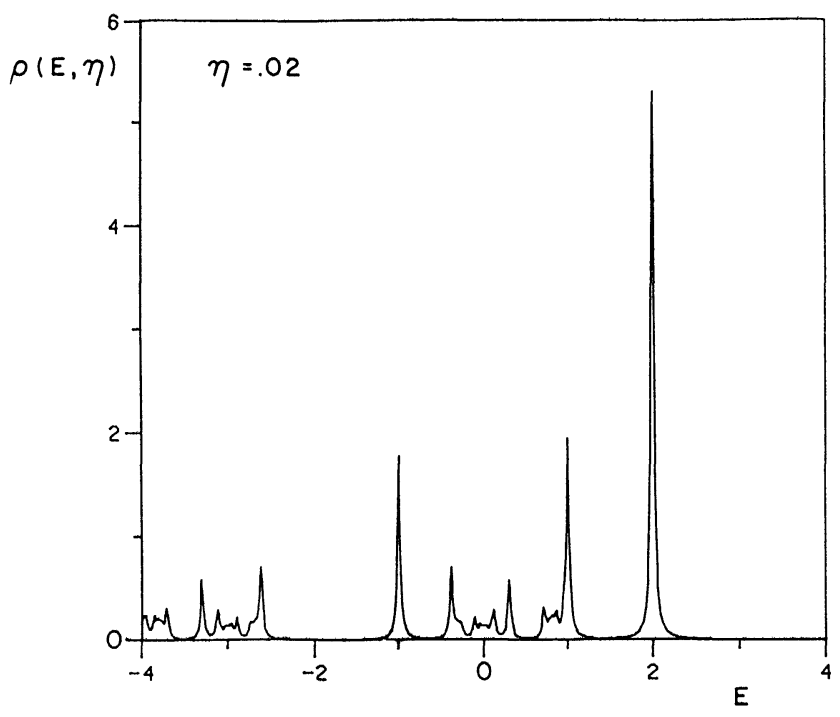

Fig. 2. - Density of states $\rho(E, \eta)$ for $\eta=0.02$ as a function of $E$ for the lattice of figure 1 . The parameters in equation 1 are taken as $\varepsilon=0, V=1$.

illustrated in figure 2 when $\eta=0.02$. It may easily be checked that the DOS integrates to unity by using the following identity [17], which rests on the analytic properties of Green's functions,

$$
\lim _{\eta \rightarrow 0} \int_{E_{\mathbf{L}}}^{E_{\mathbf{R}}} \rho(E, \eta) \mathrm{d} E=\operatorname{Im} \oint\left[\frac{1}{N} \frac{2}{\pi} \frac{\partial \mathcal{F}(z)}{\partial z}\right] \mathrm{d} z
$$

where $E_{\mathrm{L}}$ and $E_{\mathrm{R}}$ are respectively to the left and to the right of the region where states may be allowed. It is convenient to choose a contour $\mathrm{C}$ which leaves the real axis at $E_{\mathrm{L}}$, moves parallel to it at a distance $\eta$ above and then returns on the real axis at $E_{\mathrm{R}}$.

\section{Analytical proof of scaling.}

To prove scaling, as in critical phenomena, one first notes that the generating function may be expressed as a function of only the ratio $V /(E+i \eta-\varepsilon)$ instead of each quantity separately [18]. For that, it suffices to define $v_{i}=(E+i \eta-\varepsilon)^{1 / 2} u_{i}$ and to express the integrals in (2) as a function of these variables. The Jacobian of this transformation adds a $v_{i}$ independent term to $\mathcal{F}$. After each decimation, one performs such a change of variables to keep the coefficient of the quadratic terms $u_{i}^{2}$ constant. Defining $N C^{(l)}$ as the constant term in $\mathcal{F}$ after $l$ iterations, one obtains the following recursion relations

$$
\begin{aligned}
& C^{(l+1)}=C^{(l)}-\frac{1}{2} \frac{1}{3^{l+1}}\left\{\frac{1}{3} \ln \left(\frac{1-x^{(l)}}{1+2 x^{(l)}}\right)+\ln \left(\left(1-2 x^{(l)}\right)\left(1+3 x^{(l)}\right)\right)\right\} \\
& x^{(l+1)}=-\frac{x^{(l)^{2}}}{1+3 x^{(l)}}
\end{aligned}
$$

where $x^{(l)}=V^{(l)} /\left(E+i \eta-\varepsilon^{(l)}\right)$ and $C^{(0)}=-\frac{1}{2} \ln (E+i \eta)$. If after $n$ iterations all sites of the lattice have been eliminated, then $\mathcal{F}=N C^{(n)}$. For the periodic boundary conditions illustrated in figure 1 the last iteration is identical to the others. In general, for a given number of sites $N$, 
one can choose $\eta$ such that $\mathcal{F}$ converges to a required accuracy before the boundaries are reached. The integrated DOS as calculated from (7) will be unity for any value of $\eta$, but to be able to resolve the individual levels (at $N$ fixed), $\eta$ must be small enough. The DOS calculated from equations 8 , 9 and 3 is the same as that of figure 2 . Note is passing that numerical differentiation can be avoided by using instead the chain rule to compute the DOS. We have used the latter procedure, which is analogous to what is done in critical phenomena to compute the magnetization [6].

Scaling occurs close to fixed points or limit cycles of the recursion relation 9 . Indeed, we have just argued that for the Sierpińsky gasket one may write,

$$
\mathcal{F}=N^{(l)} f\left(x^{(l)}\right)+N C^{(l)}
$$

where $f\left(x^{(l)}\right)$ is the generating function per non-integrated degree of freedom. The number of such sites is $N^{(l)}=N /\left(b^{d}\right)^{l}$ where $b$ is the length rescaling factor and $\bar{d}$ the fractal dimension. $b=2$ and $b^{\bar{d}}=3$ for the lattice of figure 1 . Close to a fixed point, such as $x^{*}=-0.25$ (i.e. $E^{*}=-4$, $\eta^{*}=0$ ) one has

$$
\mathcal{F}=N f\left(x^{(0)}-x^{*}\right)+N C^{(0)}=N^{(1)} f\left(x^{(1)}-x^{*}\right)+N C^{(1)} .
$$
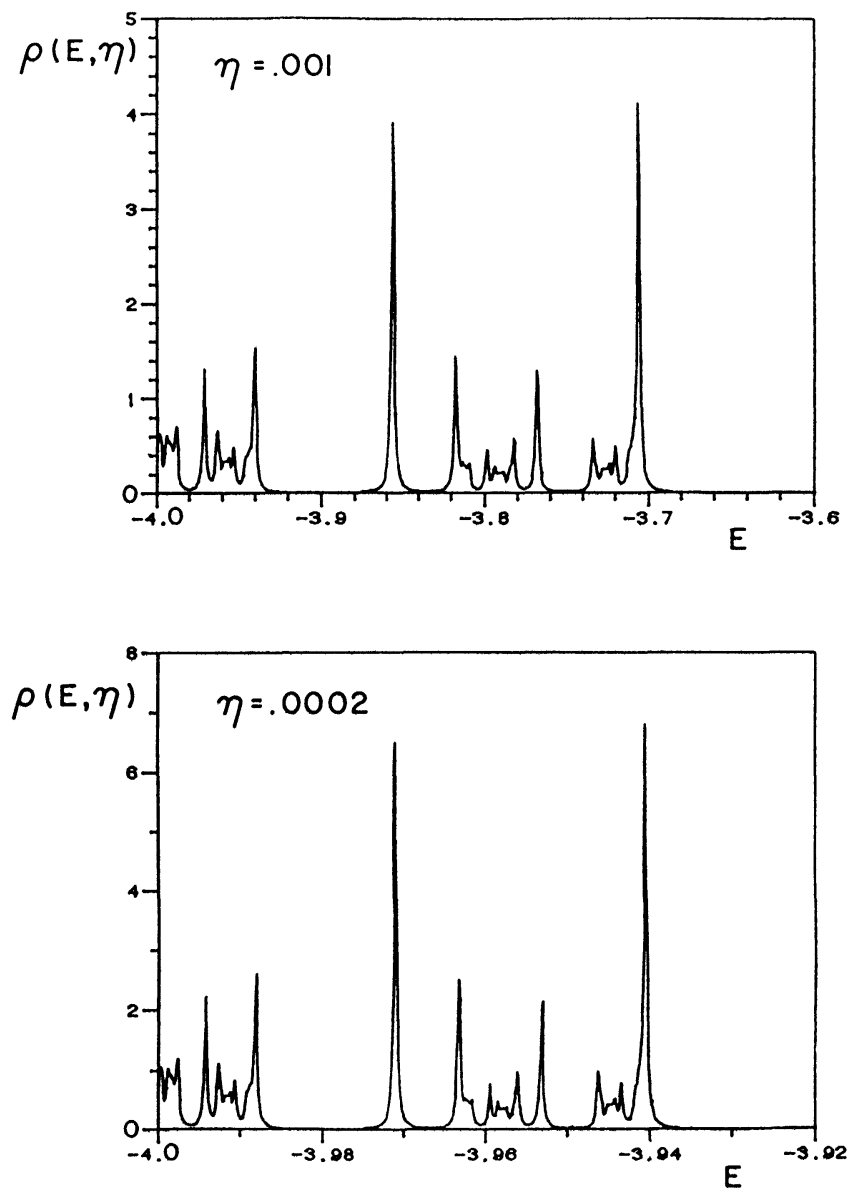

Fig. 3. - Scaling of the density of states $\rho(E, \eta)$ close to the fixed point, $E=-4$ for the lattice of figure 1 . a) $\eta=0.001$; b) $\eta=0.0002$. Note that the horizontal axis in $b$ ) is five times smaller and the density of states a factor $5 / 3$ larger, as predicted by equation 14 . 
One can check explicitly that $C^{(1)}$ and $C^{(0)}$ have negligible imaginary part close to that fixed point. The DOS there may thus be computed from $\partial f / \partial E$ only. Hence one may write from (11),

$$
\frac{\partial f}{\partial E}\left(x^{(0)}-x^{*}\right)=\frac{1}{b^{d}} \frac{\partial f}{\partial E}\left(x^{(1)}-x^{*}\right) .
$$

Using $x^{(0)}-x^{*} \equiv-\left(E+i \eta-E^{*}\right) / E^{* 2}$ and $\left(x^{(1)}-x^{*}\right) \sim b^{a}\left(x^{(0)}-x^{*}\right)$ where here $b^{a}=5$, one has,

$$
\frac{\partial f}{\partial E}\left(E+i \eta-E^{*}\right)=b^{a-\bar{a}} \frac{\partial f}{\partial^{\prime}\left(b^{a} E\right)}\left(b^{a}\left(E+i \eta-E^{*}\right)\right)
$$

and hence from (3),

$$
\rho(E, \eta)=b^{a-\bar{d}} \rho\left(b^{a} E, b^{a} \eta\right)
$$

This scaling is illustrated in figure 3. It is in agreement with the results of Rammal and Toulouse [9]. If one notes that when $\eta$ is not scaled by $b^{\iota}$ one obtains approximately

$$
\rho(E, \eta) \equiv b^{-\bar{d}} \rho\left(b^{a} E, \eta\right)
$$

then one recovers the scaling of Domany et al. [10]. As is clear from figure 3 and as pointed out before $[19,9,10]$, one has scale similarity but not power law behaviour here, because the scaling $b$ cannot be taken as arbitrary. Because the map in equation 8 may also have limit cycles, one can also get scaling behaviour around points which belong to those limit cycles [10]. Finally, it is interesting to note that the sharp structure in the DOS is in some sense only a reflection of the singular nature of the generating function (2) when it is continued on the real axis (i.e. $\eta=0$ ). There, the problem becomes a pure statistical mechanics problem analogous to the one analysed in detail by Derrida et al. [19]. Note that, as pointed out by others [9, 10], when the recursion relations are derived for the variable $w^{(l)}=\left(4+1 / x^{(l)}\right) / 5$ then equation 9 becomes

$$
w^{(l+1)}=\lambda w^{(l)}\left(1-w^{(l)}\right)
$$

with $\lambda=5$. The properties of this map in the complex plane have been studied by Mandelbrot [20].

The spectrum for the generalization of the Sierpinsky gasket in Euclidean $d=3$ is shown in figure 4. It is relatively easy to obtain using the technique just described. The scaling is consistent with previous results [9-12]. However, this is the first time this spectrum is explicity exhibited. (See also Ref. 12.)

\section{Density of states and spectral dimension of Berker-Ostlund hierarchical lattices.}

Rammal and Toulouse [9] have argued that the beta function for the conductance should change sign at $\widetilde{d}=2$, indicating a change in the localization behaviour of eigenstates. They thus suggested that a study of a class of fractal lattices whose spectral dimension can vary from $\widetilde{d}<2$ to $\tilde{d}>2$ might shed some light on the problem of localization. Unfortunately, as they also noted, Sierpińsky gaskets and their generalization to higher dimension always have $\tilde{d}<2$. We now discuss a class of lattices which in one limit have $\tilde{d}>2$ and in another can have continuously varying $\tilde{d}$.

Consider the vibrational problem on the self-similar structure whose construction is illustrated in figure 5. It was shown earlier [13] that the exact local DOS on the central site of such a lattice is the same as the one which was derived by Gonçalves da Silva and Koiller [21] from an approximate decimation scheme for the disordered binary harmonic chain [22]. We further introduce the 


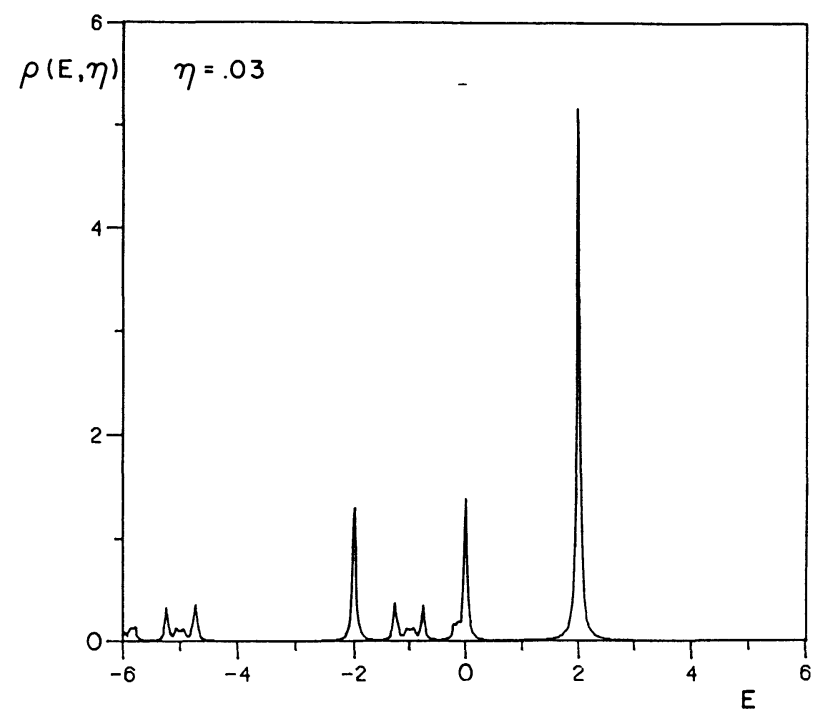

Fig. 4. - Density of states $\rho(E, \eta)$ for the Sierpińsky « web », i.e., the generalization of the lattice of figure 1 to an embedding Euclidean space of dimension 3. $\varepsilon=0, V=1$ and $\eta=0.03$.

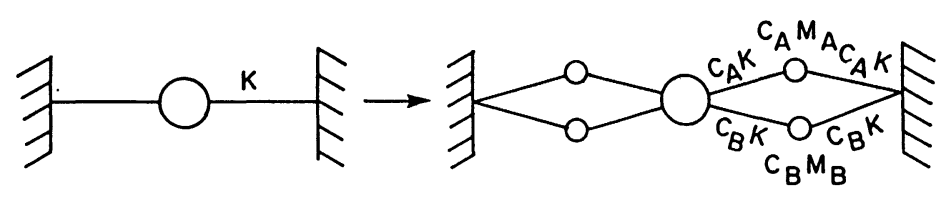

(a)

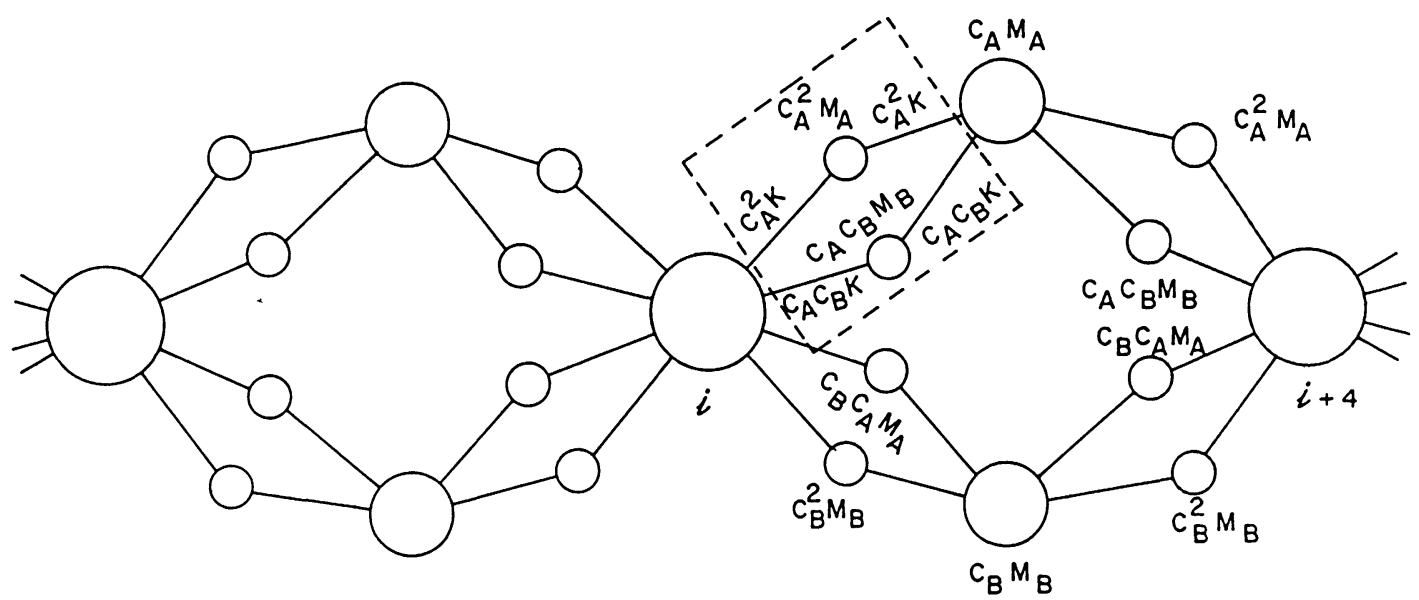

( b )

Fig. 5. - Construction of a hierarchical lattice for a mass and spring problem. a) Each spring of force constant $K$ is replaced by two masses each attached in parallel by two springs whose force constant is as indicated on the figure. In the disordered binary chain problem this lattice can model (see Ref. 13) $c_{\mathrm{A}}$ and $c_{\mathrm{B}}$ are the concentration respectively of the « primitive " masses $M_{\mathrm{A}}$ and $M_{\mathrm{B}}\left(c_{\mathrm{A}}+c_{\mathrm{B}}=1\right) \cdot \omega^{2}$ in the text is the square of the angular vibration frequency for this problem. $b$ ) Lattice obtained by applying twice the rule described in $a$ ). 
lattice of figure 6 which is a generalization of the hierarchical lattice of figure 5. One finds that there are as many scaling fields

$$
y_{i}^{(l)}=\left(2 K^{(l)}-M_{i}\left(\omega^{2}+i \eta\right)\right) /\left(2 L^{(l)}\right)
$$

as there are " primitive " masses $M_{i}=M_{\mathrm{A}}, M_{\mathrm{B}}, M_{\mathrm{C}} \ldots L^{(l)}$ is the renormalized nearest-neighbour force constant while $\left[2 L^{(l)}-\left(2 K^{(l)}-M_{i}\left(\omega^{2}+i \eta\right)\right)\right] / M_{i}$ is the renormalized frequency squared $\left(L^{(0)}=K^{(0)}=K\right)$. In the limit where the " primitive " masses are all equal, there is only one scaling variable whose recursion relation becomes the same as (16) with $\lambda=4$ when the change of variable $w^{(l)}=\frac{1}{2}\left(1-y^{(l)}\right)$ is performed. The DOS of the central site is then exactly that of the pure $d=1$ chain but the total DOS for the lattice of figure 5 , as calculated from the generating function, is shown in figure 7 and is very different from that of the chain. It has the same scaling behaviour as the electronic tight-binding model described in reference 10 . In the latter model however, only the hopping matrix element was inhomogeneous [23].

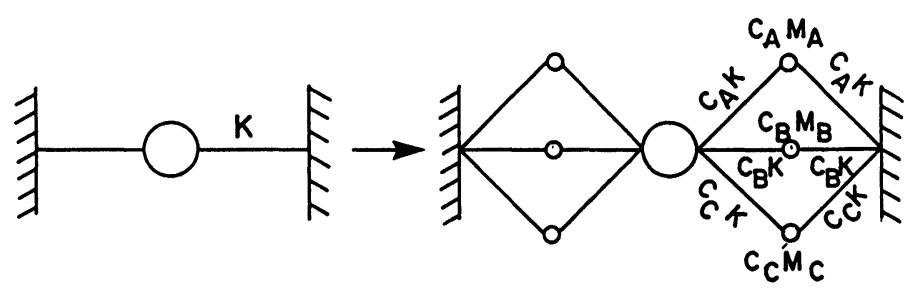

Fig. 6. - Elementary step to construct a generalization of the hierarchical lattice of figure 5. $c_{\mathrm{A}}+c_{\mathrm{B}}+$ $c_{\mathrm{C}}=1$. The case where $M_{\mathrm{A}}=M_{\mathrm{B}}=M_{\mathrm{C}}$ is discussed in the test.

Still in the case where all " primitive " masses are equal and there is a single scaling field, one finds from the generating function that

$$
\rho\left(\omega^{2}, \eta\right)=\frac{\lambda}{m} \rho\left(\lambda \omega^{2}, \lambda \eta\right)
$$

where $m$ is the number of bonds which collapse into one bond at each iteration : $m=4$ in figure 5 and $m=6$ in figure 6 . Note that to improve the analogy with genuine fractal lattices such as the Sierpińsky gasket, we consider only lattices with a very large number of sites because it is only in that limit that the number of sites scales by a factor of $m$ at each iteration like the number of bonds.

Normally, one defines $z$ and the fractal dimension $\bar{d}$ by the relations $\lambda=b^{z}$ and $m=b^{d}$. The length rescaling factor $b$ here may seem a bit ambiguous because the lattice is so inhomogeneous [24]. This inhomogeneity may in fact raise some questions as to whether this class of lattices can be properly called fractals. Nevertheless, their self-similarity and the fact that $d, \bar{d}$ and $\tilde{d}$ are in general different whatever $b$ we choose, suggest that such lattices be included in the " fractal » category.

It is important to note that the spectral dimension $\mathscr{d}=2 \bar{d} / z=2 \ln m / \ln \lambda=\ln m / \ln 2$ is independent of the choice of $b$. For $m \geqslant 4$ one finds that $\tilde{d} \geqslant 2$ providing a class of lattices which is in a sense complementary to the Sierpińsky gasket. Note that $\bar{d}=\tilde{d}$ if one makes the natural choice [24] $b=2$. The fractal dimension $\bar{d}$ apparently does not play an important rôle in Rammal and Toulouse's [9] conjecture on localization.

Lastly, we consider the case of a continuously varying $\not{d}$ for Berker-Ostlund lattices. This is obtained in the limit where one has two kinds of « primitive » masses : one group of masses, say $M$, which are all equal and finite, and another group where all masses, say $M_{\infty}$, are equal and infi- 


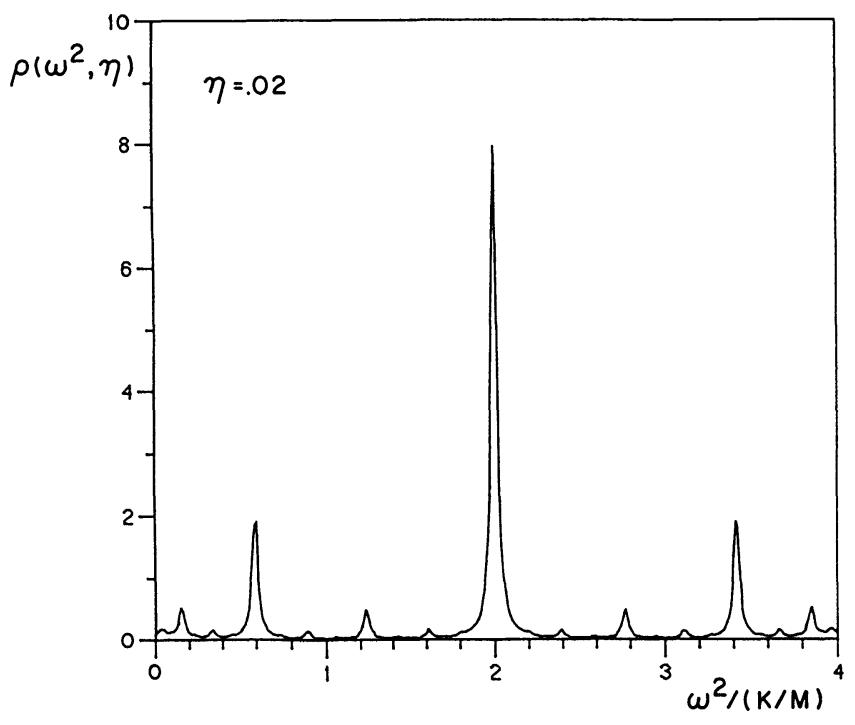

Fig. 7. - Total densty of states $\rho\left(\omega^{2}, \eta\right)(\eta=0.02)$ for the hierarchical lattice of figure 5 (in the limit $\left.M_{\mathrm{A}}=M_{\mathrm{B}}\right)$ as a function of $\omega^{2} /(K / M)$.

nite. Then, if one is interested in the spectrum everywhere but at frequencies smaller than about $\sqrt{K / M_{\infty}}$ then one needs only one scaling field, $y^{(l)}=\left(2 K^{(l)}-M\left(\omega^{2}+i \eta\right)\right) /\left(2 L^{(l)}\right)$. Defining $c$ as the total concentration of atoms with light masses, and $\lambda=1+(1+B / c)^{1 / 2}$ one finds that the recursion relation for $w^{(l)}=\frac{1}{2}\left(1-4 y^{(l)} /(\lambda c)\right)$ is the same as equation 16 . The problems considered previously in reference 10 all involved recursion relations of the form of (16) but with $\lambda \geqslant 4$ and integer. We have here an example where the value of $\lambda$ may be varied continuously above $\lambda=4$.

Like above, we find for the $b$ independent spectral dimension, $\tilde{d}=2 \ln m / \ln \lambda$. With $m=2 k$, $(k=2,3,4 \ldots)$ and $\lambda \geqslant 4$, we find that $\tilde{d}$ can be less than or larger than 2 , crossing the value 2 at $c=8 /(m(m-2))$. For $m \geqslant 6$ it is possible to have closed loops of light masses by choosing two or more of the " primitive " light masses as equal.

\section{Acknowledgments.}

We wish to acknowledge a most stimulating conversation with Y. Shapir who also pointed out reference 9. We also wish to thank $M$. Rubinstein for bringing reference 10 to our attention and M. O. Robbins for mentioning the existence of equation 7 to us. We are pleased to thank R. Rammal for later discussions which very much influenced section three of this paper and for communicating references 11 and 12 to us prior to publication. The support of the National Research and Engineering Research Council of Canada through individual operating grants and through the program " Attaché de recherche " (A.-M.S.T.) is gratefully acknowledged.

\section{References}

[1] Mandelbrot, B. B., Fractals : Form, Chance and Dimension (Freeman, San Francisco, Cal.) 1977.

[2] Bernasconi, J., Schneider, W. R., Weismann, H. J., Phys. Rev. B 16 (1977) 5250 ;

Gefen, Y., Aharony, A., Mandelbrot, B. B., KirkPatrick, S., Phys. Rev. Lett. 47 (1981) 1771.

[3] Nelson, D. R., Fisher, M. E., Ann. Phys. 91 (1975) 226 ; Dhar, D., J. Math. Phys. 18 (1977) 577. 
[4] Gefen, Y., Mandelbrot, B. B., Aharony, A., Phys. Rev. Lett. 45 (1980) 855.

[5] Berker, A. N., Ostlund, S., J. Phys. C 12 (1979) 4961 ;

Griffiths, R. B., Kaufman, M., Phys. Rev. B 26 (1982) 5022 ;

Zalys, E., Comm. Math. Phys. 67 (1979) 17 ;

MeLrose, J. R., unpublished.

[6] Neimeijer, Th., van Leeuwen, J. M. J., in Phase Transitions and Critical Phenomena, ed. by C. Domb and M. S. Green (Academic, London) vol. 6.

[7] Alexander, S., Orbach, R., J. Physique Lett. 43 (1982) L-625.

[8] Rammal, R., Toulouse, G., Phys. Rev. Lett. 49 (1982) 1194.

[9] Rammal, R., Toulouse, G., J. Physique Lett. 44 (1983) L-13.

[10] Domany, E., Alexander, S., Bensimon, D., Kadanoff, L. P., to be published.

[11] Rammal, R., J. Phys. C. (in press) plus one other unpublished work.

[12] After we first submitted this paper, we learned from R. Rammal that he has independently calculated the spectrum and eigenfunctions of Sierpińsky gaskets in arbitrary dimensions (previous reference). He also told us that he has done numerical calculations identical to ours.

[13] Langlois, J.-M., Tremblay, A.-M. S., Southern, B. W., Phys. Rev. B 28 (1983) 218.

[14] Southern, B. W., Kumar, A. A., Loly, P. D., Tremblay, A.-M. S., Phys. Rev. B 27 (1983) 1405.

[15] Edwards, S. F., Jones, R. C., J. Phys. A 9 (1976) 1595 ;

Nitzan, A., Freed, K. F., Cohen, M. H., Phys. Rev. B 15 (1977) 4476.

[16] Note that in one dimension, the generating function becomes, within constant factors, the HerbertJones-Thouless localization function defined in the following references : HERBERT, D. C., JONES, R., J. Phys. C 4 (1971) 1145 ; Thouless, D. J., J. Phys. C 5 (1972) 77.

[17] RobBINs, M. O., private communication.

[18] The inverse of this ratio, i.e. $(E+i \eta-\varepsilon) / V$ may also be used but it iterates to large values. It is thus more convenient numerically to iterate $V /(E+i \eta-\varepsilon)$.

[19] Derrida, B., Eckmann, J.-P., Erzan, A., J. Phys. A 16 (1983) 893.

[20] Mandelbrot, B. B., in " Nonlinear Dynamics Conference", Ann. N.Y. Acad. Sci. 357 (1980) 249.

[21] Gonçalves da Silva, C. E. T., Kolller, B., Solid State Commun. 40 (1981) 215.

[22] We have here an example of a highly inhomogeneous but ordered lattice which may serve as a model for homogeneously disordered systems (Ref. 13). Similar relations between approximate rescaling schemes and corresponding exact realizations on hierarchical lattices exist in general for problems with Gaussian generating functions (harmonic vibrations, random walk, etc...). (Tremblay, A.-M. S., unpublished).

[23] Berker-Ostlund lattices must be inhomogeneous to be able to find a single recursion relation.

[24] In some sense, the notion of distance is ambiguous for all spring and mass problems since springs can have different lengths and identical strength. The definition of $b$ should relate more to the labelling of sites. In this case, $b=2$ is the most natural definition for the rescaling we perform on lattices of the type illustrated in figures 5 and 6. 